\title{
FROBENIUS GROUPS AS MONODROMY GROUPS
}

\author{
ROBERT M. GURALNICK \\ (Received 3 February 2008; accepted 4 April 2008) \\ Communicated by Martin W. Liebeck \\ Dedicated to Cheryl Praeger for her sixtieth birthday
}

Abstract

We study Frobenius groups acting on curves.

2000 Mathematics subject classification: primary 14H30, 14H05; secondary 12F10, $20 \mathrm{~B} 25$. Keywords and phrases: Frobenius group, monodromy group, coverings of curves.

\section{Introduction}

Let $k$ be an algebraically closed field of characteristic $p \geq 0$. Consider a separable nontrivial rational map $f: X \rightarrow Y$ between smooth projective curves $X, Y$ defined over $k$. We call the Galois group of the Galois closure of $k(X) / k(Y)$ the monodromy group of $f$. A major tool in studying such covers is to translate arithmetic and geometric questions to questions about the monodromy group. This has been used very successfully in many instances. See [4] and [5] for examples and other references.

Recall that a Frobenius group is a finite permutation group $G$ acting transitively on a set $\Omega$ with nontrivial point stabilizer such that no nonidentity element fixes two points. It follows that there is a Frobenius kernel $N$, a normal subgroup such that $N^{\#}=N \backslash(1)$ is precisely the set of fixed point free elements of $G$, and a Frobenius complement $H$ (a point stabilizer). Rather surprisingly the only proof that the Frobenius kernel exists involves character theory (this was first proved by Frobenius).

This implies easily that $N$ acts regularly on $\Omega$. So we can identify $\Omega$ with $N$ as an $H$-set, and so every nontrivial element of $H$ acts on $N^{\#}$ by conjugation without fixed points. By a famous theorem of Thompson [7], this implies that $N$ is nilpotent.

A rational function is a map from $\mathbb{P}^{1}$ to $\mathbb{P}^{1}$; similarly, a polynomial is a rational function that is totally ramified at $\infty$.

The author was partially supported by NSF Grant DMS 0653873.

(c) 2008 Australian Mathematical Society 1446-7887/08 \$A2.00+0.00 
In this note, we show that rational functions with monodromy group a Frobenius group have very special properties; in particular, the Galois closure has genus at most one. This was originally proved independently by the author [2] and Flynn [1]. These come up in many of the examples of interesting polynomials (for example, exceptional polynomials, subadditive polynomials) and also come up in a reduction theorem of the author (see $[4,5,3])$. The proofs given here are representation theoretic in nature and quite different from the earlier proofs.

In fact, we prove a much more general result for Frobenius groups acting on a curve $X$; see Theorem 3.1 for the precise statement. We also prove an analog under a weaker condition on fixed points of elements in inertia subgroups (see Theorem 4.2).

See [4] or [5] for basic results on monodromy groups and coverings of curves.

\section{Basic properties of frobenius groups}

We first point out an easy property of Frobenius groups. Recall that a group acts semiregularly on a set if no nonidentity element of the group fixes a point. If $V$ is a $G$-module, let $V^{G}$ denote the fixed points of $G$ on $V$. If $H$ is a subgroup of $G$ and $W$ is an $H$-module, let $W_{H}^{G}$ denote the induced module.

LEMMA 2.1. Let $G$ be a Frobenius group with $k$ a field.

(1) The subgroup $H$ acts semiregularly on the set of isomorphism classes of nontrivial irreducible modules of $N$ (by conjugation).

(2) If $V$ is an irreducible $k G$-module, then either $V^{N}=V$ or $V \cong W_{N}^{G}$ for some (nontrivial) irreducible $N$-module $W$.

(3) If $V$ is an irreducible $k G$-module, then either $V^{N}=V$ or $V$ is a free module for $H$ and $V^{H} \neq 0$.

Proof. Let $V$ be an irreducible $k N$-module. Suppose that $1 \neq h \in H$ preserves $V$. Let $M$ be the kernel of $N$ on $V$. Since $N$ is nilpotent, $N / M$ has a nontrivial center and $h$ must centralize this center (since it preserves the representation), whence $C_{N}(h) \neq 1$ (since the order of $h$ is coprime to $|N|$ ). This contradicts the definition of Frobenius group.

Let $V$ be an irreducible $G$-module with $V^{N} \neq V$. Let $W$ be an irreducible $N$-submodule of $V$. By (1), $W_{N}^{G}$ is a direct sum of nonisomorphic $N$-modules permuted freely by $H$ and in particular is irreducible. Since $0 \neq \operatorname{Hom}_{N}(W, V)$ $\cong \operatorname{Hom}_{G}\left(W_{N}^{G}, V\right)$ (by Frobenius reciprocity), it follows that $V \cong W_{N}^{G}$. This implies that $V$ is a free $H$-module. Parts (2) and (3) follow.

COROllary 2.2. Let $G$ be a Frobenius group with Frobenius kernel $N$ and complement $H$. Let $V$ be a finite-dimensional $\mathbb{C} G$-module with $V^{G}=0$. Then $\operatorname{dim} V=\operatorname{dim} V^{N}+|H| \operatorname{dim} V^{H}$.

PROOF. It suffices to prove this formula for an irreducible nontrivial $G$-module. If $V^{N}=V$, then $V^{H}=V^{G}=0$ since $V$ is nontrivial. If $V^{N}=0$, then $V$ is a free $H$-module, whence the result holds. 


\section{Frobenius groups acting on curves}

We first recall some facts about the Tate module for a finite group acting on a curve $X$. The Tate module is a $\mathbb{C} G$-module of dimension $2 g$ with $g$ the genus of $X$. It can be constructed as follows. Let $r$ be a prime different from the characteristic of $X$ with $r$ not dividing the order of $G$. Let $W$ be the $r$-torsion points of the Jacobian of $X$. This has order $r^{2 g}$ and is a module for $G$. Its Brauer character is the character of $G$ on the Tate module (this defines the Tate module; it does not depend upon the choice of $r$ ). The Tate module is uniquely determined by noting that its character is rational valued and that, if $H$ is a subgroup of $G$, then $\operatorname{dim} V^{H}=2 g(X / H)$. This is the property that we require. Applying Corollary 2.2 to the Tate module gives the following corollary.

COROLlary 3.1. Let $G$ be a Frobenius group acting on a curve $X$ of genus $g$ with $X / G$ of genus zero. Let $N$ be the Frobenius kernel and $H$ a Frobenius complement. Then $g=g(X / N)+g(X / H)|H|$.

The special case when $g(X / H)=0$ had been proved much earlier independently by the author and Flynn [1, Theorem 9]. The previous result with $g(X / H)=0$ says that $g=g(X / N)$. This implies that $g \leq 1$ (since if $X$ is a curve of genus $g>1$, there is no separable map of degree greater than one from $X$ to another curve of genus $g$ ). Moreover, if $g=1$, then $g(X / N)=1$, and so the cover $X \rightarrow X / N$ must be unramified (and conversely). In particular, it follows that $N$ is abelian of rank at most two. By considering subgroups of $\operatorname{Aut}\left(\mathbb{P}^{1}\right)=\operatorname{PGL}(2, k)$ and $\operatorname{Aut}(X)$ with $X$ of genus one, we have the following result (see [6] for facts about automorphism groups of elliptic curves).

COROLlary 3.2. Let $G$ be a Frobenius group acting on a curve $X$ of genus $g$ over a field $k$ of characteristic $p \geq 0$. Let $N$ be the Frobenius kernel and $H$ a Frobenius complement of index $n$. If $X / H$ has genus zero, then $g \leq 1$. Moreover, $N$ is abelian. Furthermore:

(1) either $g=0$, and

(a) $G$ is dihedral of order $2 n$, or

(b) $n=4$, or

(c) $n=p^{a}$;

(2) or $g=1, X \rightarrow X / N$ is unramified ( $X / N$ also has genus one) and $H$ is cyclic of order two, three, four or six or $p \leq 3$.

By considering the automorphism groups of curves of genus at most one, we can write down all such examples. We single out a special case.

COROllary 3.3. Let $f(x)$ be a separable rational function in $k(x)$ of prime degree $r$. Assume that $k$ is algebraically closed of characteristic $p$. Assume that the Galois group $G$ of the Galois closure L of $k(x) / k(f(x))$ is solvable. Then $G$ has a normal subgroup $N$ of order $r$ and one of the following holds: 
(1) there is a totally ramified point, L has genus zero, and

(a) $r \neq p$ and $G$ is cyclic of order $r$ or dihedral of order $2 r$, or

(b) $r=p$ and $G \leq \operatorname{AGL}(1, p)$;

(2) there is no totally ramified point, $L=k(E)$ where $E$ is an elliptic curve, $E \rightarrow E / N$ is unramified and $G / N$ is a nontrivial cyclic subgroup of $\operatorname{Aut}(E)$; in particular, $G / N$ has order two, three, four or six.

Proof. Observe that $G$ is a solvable transitive subgroup of the symmetric group of degree $r$. Thus, $G$ is a Frobenius group (or is cyclic of order $r$ ). Thus, our earlier results apply and it is straightforward to determine the possibilities.

One can easily write down the rational functions (up to equivalence) that occur in the previous result. In particular, if $r \neq p$ and $f$ is a polynomial, then $L$ has genus zero and $f$ is equivalent either to $x^{r}$ or to a Dickson polynomial of degree $r$.

\section{A variation on the theme}

Now we consider another variation. Rather than consider the case where $G$ is a Frobenius group, we just assume that:

(*) $G$ is a finite group acting on a curve $X$ of genus $g$ with a subgroup $H$ of index $n>1$. If $1 \neq x \in G$ fixes some point of $X$, then $x$ fixes at most one point on $G / H$.

So we are only assuming the condition that nontrivial elements of inertia groups fix no more than one point on $G / H$. We first point out the following result. Recall that $O_{p}(J)$ is the largest normal $p$-subgroup of $J$.

LEMma 4.1. Let $G$ be a finite transitive permutation group on the a $\Omega$ of cardinality $n$. Let $I$ be a subgroup of $G$ with $I / O_{p}(G)$ cyclic. Assume that, if $1 \neq g \in I$, then $g$ fixes at most one point on $\Omega$. Then every orbit except perhaps one is regular for $I$. In particular, the number of orbits of $I$ on $\Omega$ is at most $(n-1) /|I|+1$. Moreover, equality holds precisely when I fixes a point of $\Omega$.

PROOF. We may assume that $I$ has at least one nonregular orbit. Let $w$ be a point in that orbit, and let $x \in I$ be an element of prime order $r$ fixing $w$. Note that the centralizer of $x$ in $G$ must also fix $w$ (since $w$ is the unique point fixed by $x$ ). In particular, if $r=p$, then the center $Z$ of $O_{p}(I)$ fixes $w$ as does the normalizer. Since $w$ is also the unique fixed point of $Z$ and $I$ normalizes $Z, I$ also fixes $w$. In this case $I$ has a fixed point, and all other orbits are regular. Thus the number of orbits is $1+(n-1) /|I|$.

So we may assume that no nontrivial element of $O_{p}(I)$ fixes a point of $\Omega$ and $r \neq p$. In particular, it follows that any element of $I$ of order prime to $p$ fixes a point in $I w$, and so has no fixed points in any other $I$-orbit. In this case, there is one orbit of size $\left|O_{p}(I)\right|$ and all other orbits are regular. 
THEOREM 4.2. Assume that (*) holds. Let h be the genus of $X / H$ and $|G|=m$.

(1) Then $g-1 \leq \mathrm{hm} /(n-1)$, with equality if and only if each inertia subgroup is conjugate to a subgroup of $H$.

(2) In particular, if $h=0$, then $X$ has genus at most one. Moreover, $X$ has genus one if and only if each inertia group is conjugate to a subgroup of $H$.

PROOF. Let $g$ be the genus of $X$ and $h$ the genus of $X / G$. Let $J$ be any subgroup of an inertia group. Set $n=[G: H]$ and $m=|G|$.

By the Riemann-Hurwitz formula,

$$
2(g-1) / m=-2+\sum a_{J}(1-1 /|J|)
$$

and

$$
2(h-1) / n=-2+\sum a_{J}(1-\operatorname{orb}(J, G / H)) n .
$$

Here the sum runs over some family of subgroups each contained in an inertia group and the $a_{J}$ are positive rational numbers. Also orb $(J, G / H)$ is the number of orbits of $J$ on $G / H$. By the previous lemma, orb $(J, G / H) \leq 1+(n-1) /|J|$ and so

$$
1-\operatorname{orb}(J, G / H) / n \geq(n-1) / n-(n-1) / n|J|=[(n-1) / n](1-1 / J \mid) .
$$

Thus, multiplying the second equation by $n /(n-1)$ and using equality in the third equation, we see that

$$
2(h-1) /(n-1) \geq-2 n /(n-1)+\sum a_{J}(1-1 /|J|)=2(g-1) / m-2 /(n-1) .
$$

So $h /(n-1) \geq(g-1) / m$ or $g-1 \leq h m /(n-1)$. In particular, $h=0$ implies that $g \leq 1$. The same argument shows that we have a strict inequality above unless each inertia group has one orbit of size one and all other orbits regular (and in this case, we have equality, forcing $g=1$ ).

\section{References}

[1] John Flynn, 'Near-exceptionality over finite fields', PhD Thesis, University of California Berkeley, 2001.

[2] Robert M. Guralnick, 'Rational functions with monodromy group a Frobenius group', Preprint, 2000 .

[3] - 'Solvable monodromy groups of low genus covers', Preprint.

[4] - Monodromy Groups of Coverings of Curves, Galois Groups and Fundamental Groups, Mathematical Sciences Research Institute Publications, 41 (Cambridge University Press, Cambridge, 2003), pp. 1-46.

[5] Robert M. Guralnick, Peter Müller and Jan Saxl, 'The rational function analogue of a question of Schur and exceptionality of permutation representations', Mem. Amer. Math. Soc. 162(773) (2003).

[6] Joseph H. Silverman, The Arithmetic of Elliptic Curves, Graduate Texts in Mathematics, 106 (Springer, New York, 1992).

[7] J. G. Thompson, 'Finite groups with fixed-point-free automorphisms of prime order', Proc. Natl. Acad. Sci. USA 45 (1959), 578-581. 
ROBERT M. GURALNICK, Department of Mathematics, University of Southern California, Los Angeles, CA 90089-2532, USA e-mail: guralnic@usc.edu 